\title{
On Two Conjectures on Packing of Graphs
}

\author{
BÉLA BOLLOBÁS, ${ }^{1 \dagger}$ ALEXANDR KOSTOCHKA ${ }^{2 \ddagger}$ \\ and KITTIKORN NAKPRASIT \\ ${ }^{1}$ University of Memphis, Memphis, TN 38152, USA \\ and \\ Trinity College, Cambridge CB2 1TQ, UK \\ (e-mail: bollobas@msci.memphis.edu) \\ ${ }^{2}$ University of Illinois, Urbana, IL 61801, USA \\ and \\ Institute of Mathematics, Novosibirsk 630090, Russia \\ (e-mail: kostochk@math.uiuc.edu) \\ ${ }^{3}$ University of Illinois, Urbana, IL 61801, USA \\ (e-mail: nakprasi@math.uiuc.edu)
}

Received 16 January 2004; revised 1 September 2004

For Béla Bollobás on his 60th birthday

In 1978, Bollobás and Eldridge [5] made the following two conjectures.

(C1) There exists an absolute constant $c>0$ such that, if $k$ is a positive integer and $G_{1}$ and $G_{2}$ are graphs of order $n$ such that $\Delta\left(G_{1}\right), \Delta\left(G_{2}\right) \leqslant n-k$ and $e\left(G_{1}\right), e\left(G_{2}\right) \leqslant c k n$, then the graphs $G_{1}$ and $G_{2}$ pack.

(C2) For all $0<\alpha<1 / 2$ and $0<c<\sqrt{1 / 8}$, there exists an $n_{0}=n_{0}(\alpha, c)$ such that, if $G_{1}$ and $G_{2}$ are graphs of order $n>n_{0}$ satisfying $e\left(G_{1}\right) \leqslant \alpha n$ and $e\left(G_{2}\right) \leqslant c \sqrt{n^{3} / \alpha}$, then the graphs $G_{1}$ and $G_{2}$ pack.

Conjecture (C2) was proved by Brandt [6]. In the present paper we disprove (C1) and prove an analogue of $(\mathrm{C} 2)$ for $1 / 2 \leqslant \alpha<1$. We also give sufficient conditions for simultaneous packings of about $\sqrt{n} / 4$ sparse graphs.

\section{Introduction}

One of the basic notions of graph theory is that of packing. Two graphs, $G_{1}$ and $G_{2}$, of the same order are said to pack if $G_{1}$ is a subgraph of the complement $\bar{G}_{2}$ of $G_{2}$, or,

$\dagger$ Research supported by NSF grants DMS-9970404 and EIA-0130352, and DARPA grant F33615-01-C1900.

$\ddagger$ Research supported by NSF grant DMS-0099608 and by grants 99-01-00581 and 00-01-00916 of the Russian Foundation for Basic Research. 
equivalently, $G_{2}$ is a subgraph of the complement $\bar{G}_{1}$ of $G_{2}$. The study of packings of graphs was started in the 1970s by Sauer and Spencer [13] and Bollobás and Eldridge [5].

In particular, Sauer and Spencer [13] proved the following result.

Theorem 1.1. Suppose that $G_{1}$ and $G_{2}$ are graphs of order $n$ such that $2 \Delta\left(G_{1}\right) \Delta\left(G_{2}\right)<n$. Then $G_{1}$ and $G_{2}$ pack.

The main conjecture in the area is the Bollobás-Eldridge-Catlin (BEC) conjecture (see $[4,3,5,10]$ ) stating that if $G_{1}$ and $G_{2}$ are graphs with $n$ vertices, maximum degrees $\Delta_{1}$ and $\Delta_{2}$, respectively, and $\left(\Delta_{1}+1\right)\left(\Delta_{2}+1\right) \leqslant n+1$, then $G_{1}$ and $G_{2}$ pack. If true, this conjecture is a considerable extension of the Hajnal-Szemerédi theorem [12] on equitable colouring, which is itself an extension of the Corrádi-Hajnal theorem on equitable 3colourings of graphs. Indeed, the Hajnal-Szemeredi theorem is the special case of the BEC conjecture when $G_{2}$ is a disjoint union of cliques of the same size [12]. The conjecture has also been proved when either $\Delta_{1} \leqslant 2[1,2]$, or $\Delta_{1}=3$ and $n$ is huge [11]. The progress on the topic has been surveyed by Yap [16] and Wozniak [15].

The following two theorems are the main results of Bollobás and Eldridge [5].

Theorem 1.2. Suppose that $G_{1}$ and $G_{2}$ are graphs with $n$ vertices, $\Delta\left(G_{1}\right), \Delta\left(G_{2}\right)<n-$ $1, e\left(G_{1}\right)+e\left(G_{2}\right) \leqslant 2 n-3$ and $\left\{G_{1}, G_{2}\right\}$ is not one of the following pairs: $\left\{2 K_{2}, K_{1} \cup K_{3}\right\}$, $\left\{\bar{K}_{2} \cup K_{3}, K_{2} \cup K_{3}\right\},\left\{3 K_{2}, \bar{K}_{2} \cup K_{4}\right\},\left\{\bar{K}_{3} \cup K_{3}, 2 K_{3}\right\},\left\{2 K_{2} \cup K_{3}, \bar{K}_{3} \cup K_{4}\right\},\left\{\bar{K}_{4} \cup K_{4}, K_{2} \cup\right.$ $\left.2 K_{3}\right\},\left\{\bar{K}_{5} \cup K_{4}, 3 K_{3}\right\}$. Then $G_{1}$ and $G_{2}$ pack.

Theorem 1.3. For $0<\alpha<1 / 2$, there is an integer $n_{0}=n_{0}(\alpha)$ such that, if $G_{1}$ and $G_{2}$ are graphs of order $n \geqslant n_{0}$ with $e\left(G_{1}\right) \leqslant \alpha$ and $e\left(G_{2}\right) \leqslant \frac{1-2 \alpha}{5} n^{3 / 2}$, then $G_{1}$ and $G_{2}$ pack.

Let $n$ be even, $x$ be odd, $G_{1}(n)$ be a perfect matching on $n$ vertices and $G_{2}(n, x)$ be the complete bipartite graph $K_{x, n-x}$. Since $x$ is odd, the graphs $G_{1}(n)$ and $G_{2}(n, x)$ do not pack. Since $e\left(G_{1}(n)\right)=n / 2$ and $e\left(G_{2}(n, x)\right)=x(n-x)<x n$, these examples show that the condition $\alpha<1 / 2$ in Theorem 1.3 cannot be relaxed without imposing other restrictions on $G_{1}$ and/or $G_{2}$. However, Bollobás and Eldridge [5] could not find an example showing that the factor $(1-2 \alpha) / 5$ is close to optimal, and they were led to the following conjecture.

Conjecture 1.4. For all $0<\alpha<1 / 2$ and $0<c<\sqrt{1 / 8}$, there exists an $n_{0}=n_{0}(\alpha, c)$ such that, if $G_{1}$ and $G_{2}$ are graphs of order $n>n_{0}$ satisfying $e\left(G_{1}\right) \leqslant \alpha n$ and $e\left(G_{2}\right) \leqslant c \sqrt{n^{3} / \alpha}$, then the graphs $G_{1}$ and $G_{2}$ pack.

This conjecture was proved by Brandt [6] in 1995. As the main result of this paper, we prove the following extension of this theorem of Brandt to the case when $G_{1}$ has $\alpha n$ edges, with $1 / 2 \leqslant \alpha<1$.

Theorem 1.5. Let $1 / 2 \leqslant \alpha<1$ and $c>0$ satisfy

$$
8 \alpha c^{2}<1
$$


and put

$$
\varepsilon=\frac{1}{4} \min \left\{1-\alpha, 1-8 \alpha c^{2}\right\}
$$

Let $G_{1}$ and $G_{2}$ be graphs of order

$$
n>(10 / \varepsilon)^{6}
$$

such that $e\left(G_{1}\right) \leqslant \alpha n, e\left(G_{2}\right) \leqslant c n^{3 / 2}$, and $\Delta\left(G_{2}\right)<n-1-\frac{\sqrt{n}}{\sqrt{2 \alpha(1-\alpha)}}$. Then $G_{1}$ and $G_{2}$ pack.

Observe that the only additional restriction in Theorem 1.5 is that each vertex in $G_{2}$ has at least $\frac{\sqrt{n}}{\sqrt{2 \alpha(1-\alpha)}}$ non-neighbours. The example of $G_{1}(n)$ and $G_{2}(n, x)$ where $x$ is the largest odd integer not exceeding $c \sqrt{n}$ shows that the factor $\sqrt{n}$ is unavoidable there.

The examples of a perfect matching and $G_{2}(n, x)$ also explain why Bollobás and Eldridge [5, p. 118] made the following conjecture.

Conjecture 1.6. There exists an absolute constant $c>0$ such that, if $k \geqslant 1$ and $G_{1}$ and $G_{2}$ are graphs of order $n$ satisfying the conditions $\Delta\left(G_{1}\right), \Delta\left(G_{2}\right) \leqslant n-k$ and $e\left(G_{1}\right), e\left(G_{2}\right) \leqslant c k n$, then the graphs $G_{1}$ and $G_{2}$ pack.

We shall disprove Conjecture 1.6; more precisely, we shall prove the following result.

Theorem 1.7. Let $k$ be a positive integer and $q$ be a prime power. Then for every $n \geqslant$ $q \frac{q^{k+1}-1}{q-1}$, there are graphs $G_{1}(n, k)$ and $G_{2}(n, q, k)$ of order $n$ that do not pack and have the following properties:

(a) $G_{1}(n, k)$ is a forest with $n-k$ edges and maximum degree at most $n / k$;

(b) $G_{2}(n, q, k)$ is a $\frac{q^{k}-1}{q-1}$-degenerate graph with maximum degree at most $2 n / q$.

Theorem 1.7 not only disproves Conjecture 1.6, but also shows that Theorem 1.5 can not be extended even to $\alpha=1$ without essential restrictions on the maximal degree of $G_{2}$.

The rest of the paper is organized as follows. In the next section we shall discuss properties of special enumerations of vertices in graphs; our proof of Theorem 1.5, which is to be given in Section 3, will be based on these enumerations. In Section 4 we shall make use of the proof of Theorem 1.5 to give conditions providing simultaneous packing of about $\frac{1}{4} \sqrt{n / \alpha^{3}}$ graphs of order $n$ with at most $\alpha n$ edges each. More precisely, we shall prove the following result.

Theorem 1.8. Let $\frac{1}{2} \leqslant \alpha<1$,

$$
n>(50 /(1-\alpha))^{6}
$$

and $m=\left\lceil\frac{1}{4} \sqrt{n / \alpha^{3}}\right\rceil$. Let $H_{1}, H_{2}, \ldots, H_{m}$ be graphs with $n$ vertices and at most an edges each. Then $H_{1}, H_{2}, \ldots, H_{m}$ pack.

In the final section, Section 5, we discuss counterexamples to Conjecture 1.6 and prove Theorem 1.7 . 
Note that the proofs of upper bounds are algorithmic, and so enable one to construct polynomial-time algorithms for packing graphs satisfying the conditions of Theorems 1.5 or 1.8 .

\section{Greedy and degenerate enumerations}

Before embarking on the proof of Theorem 1.5, we introduce some notation and prove some auxiliary statements.

Let $v_{1}, v_{2}, \ldots, v_{n}$ be an enumeration of the vertices of a graph $G$. For $1 \leqslant i \leqslant n$, let $G(i)$ be the subgraph of $G$ induced by the vertices $v_{i}, v_{i+1}, \ldots, v_{n}$; thus $G(1)=G$ and $G(n)$ consists of the single vertex $v_{n}$. We call $v_{1}, v_{2}, \ldots, v_{n}$ a greedy enumeration of the vertices or, somewhat loosely, a greedy order on $G$, if $d_{G(i)}\left(v_{i}\right)=\Delta(G(i))$ for every $i, 1 \leqslant i \leqslant n$, i.e., the vertex $v_{i}$ has maximal degree in $G(i)$. Similarly, the enumeration and order are degenerate if $d_{G(i)}\left(v_{i}\right)=\delta(G(i))$ for every $i, 1 \leqslant i \leqslant n$, i.e., the vertex $v_{i}$ has minimal degree in $G(i)$. Note that if $v_{1}, v_{2}, \ldots, v_{n}$ is a greedy order on $G$ then $v_{i}, v_{i+1}, \ldots, v_{n}$ is a greedy order on $G(i)$, and an analogous assertion holds for the degenerate order. Another simple observation is that $v_{1}, v_{2}, \ldots, v_{n}$ is a greedy order on $G$ if and only if it is a degenerate order on the complement $\bar{G}$. Needless to say, a graph may have numerous greedy orders and degenerate orders.

For a graph $G$, set

$$
\varphi(G)=\sum_{v \in V(G)} \frac{1}{1+d_{G}(v)} .
$$

The result below is a slight extension of an inequality due to Caro [7] and Wei [14], first published in [8], implying a weak form of Turán's theorem. We formulate it in the usual way, for the complement of the graph, i.e., for finding a large independent set rather than a complete subgraph.

Theorem 2.1. Let $v_{1}, v_{2}, \ldots, v_{n}$ be a greedy enumeration of the vertices of a graph $G$, and set $\ell=\lceil\varphi(G)\rceil$. Then the last $\ell$ vertices form an independent set. Equivalently, if $d_{G(i)}\left(v_{i}\right) \geqslant 1$ then $G(i)$ has an independent set of at least $\varphi(G)$ vertices.

Proof. We apply induction on the number of edges of $G$. If there are no edges then $\varphi(G)=n$ and the entire vertex set is independent, as required. Suppose that $G$ has $m>0$ edges and the result holds for graphs with fewer edges. Write $d$ for the maximal degree of $G$, i.e., for the degree of $v_{1}$, and let $u_{1}, u_{2}, \ldots, u_{d}$ be the neighbours of $v_{1}$. Then

$$
\begin{aligned}
\varphi(G(2)) & =\varphi(G(1))-\frac{1}{d+1}+\sum_{i=1}^{d}\left(\frac{1}{d\left(u_{i}\right)}-\frac{1}{d\left(u_{i}\right)+1}\right) \\
& =\varphi(G(1))-\frac{1}{d+1}+\sum_{i=1}^{d} \frac{1}{d\left(u_{i}\right)\left(d\left(u_{i}\right)+1\right)} \\
& \geqslant \varphi(G(1))-\frac{1}{d+1}+d \frac{1}{d(d+1)}=\varphi(G(1))=\varphi(G) .
\end{aligned}
$$


By the induction hypothesis, the last $\lceil\varphi(G(2))\rceil \geqslant\lceil\varphi(G)\rceil=\ell$ vertices of $v_{2}, v_{3}, \ldots, v_{n}$ form an independent set of $G(2)$, and so of $G$, completing the proof.

We shall also need the following simple but somewhat technical lemma concerning greedy orders.

Lemma 2.2. Let $\alpha, \gamma$ and $\varepsilon$ be positive numbers satisfying $\gamma \leqslant \alpha \leqslant 1-2 \varepsilon$ and $k_{0} \leqslant(1-$ $\gamma-\varepsilon / 2) n-1$ a nonnegative integer. Let $v_{1}, v_{2}, \ldots, v_{n}$ be an enumeration of the vertices of a graph $G$ with $m$ edges with the following properties:

(i) $e\left(G_{k_{0}+1}\right) \leqslant m\left(1-\frac{2 k_{0}(\alpha+\varepsilon)}{n(\alpha-\gamma+\varepsilon / 2)}\right)$;

(ii) the enumeration $v_{k_{0}+1}, v_{k_{0}+2}, \ldots, v_{n}$ is greedy.

Then there is an index $i, k_{0} \leqslant i \leqslant(1-\gamma-\varepsilon / 2) n$, such that

$$
\Delta(G(i+1))=d_{G(i+1)}\left(v_{i+1}\right)<\frac{2 m(n-i)(\alpha+\varepsilon)}{n^{2}(\alpha-\gamma+\varepsilon / 2)} .
$$

Proof. Suppose that the assertion is false. Then for $k=\lceil(1-\gamma-\varepsilon / 2) n\rceil$ we have

$$
\begin{aligned}
e(G(k+1)) & =e\left(G\left(k_{0}+1\right)\right)-\sum_{i=k_{0}+1}^{k} \Delta(G(i)) \\
& \leqslant m\left(1-\frac{2 k_{0}(\alpha+\varepsilon)}{n(\alpha-\gamma+\varepsilon / 2)}\right)-\sum_{i=k_{0}+1}^{k} \frac{2 m(n+1-i)(\alpha+\varepsilon)}{n^{2}(\alpha-\gamma+\varepsilon / 2)} \\
& \leqslant m-\sum_{i=1}^{k} \frac{2 m(n+1-i)(\alpha+\varepsilon)}{n^{2}(\alpha-\gamma+\varepsilon / 2)} \\
& =m-\frac{2 m(\alpha+\varepsilon)}{n^{2}(\alpha-\gamma+\varepsilon / 2)}\left(\left(\begin{array}{c}
n+1 \\
2
\end{array}\right)-\left(\begin{array}{c}
n+1-k) \\
2
\end{array}\right)\right) \\
& <m-\frac{m(\alpha+\varepsilon)}{n^{2}(\alpha-\gamma+\varepsilon / 2)}\left(n^{2}-(n-k)^{2}\right) \\
& \leqslant m\left(1-\frac{(\alpha+\varepsilon)\left(1-(\gamma+\varepsilon / 2)^{2}\right)}{(\alpha-\gamma+\varepsilon / 2)}\right)=\rho m,
\end{aligned}
$$

say. To arrive at a contradiction and so complete the proof, we shall show that $\rho<0$. To this end, set $\delta=\gamma+\varepsilon / 2$, and note that

$$
\rho(\alpha-\gamma+\varepsilon / 2)=\delta(\delta(\alpha+\varepsilon)-1)
$$

Since, by assumption, $\delta>0$ and

$$
\delta(\alpha+\varepsilon) \leqslant(\alpha+\varepsilon / 2)(\alpha+\varepsilon)<1,
$$

identity (2.3) implies that $\rho$ is indeed negative, completing our proof.

We shall also use the following fact observed by several authors.

Claim 2.3. Suppose that we are packing the vertices of a graph $G_{1}$ in the reverse degenerate order into (the complement of ) a graph $G_{2}$ of order $N$ and maximal degree $D_{2}$. Suppose that 
we have already packed $j$ vertices and a vertex $w \in V\left(G_{1}\right)$ has $x$ neighbours among these $j$ vertices. If

$$
j+x D_{2}<N
$$

then we can also find a legal placement for $w$.

Proof. We cannot place $w$ at the $j$ vertices of $G_{2}$ that we have already used and into $G_{2}$-neighbours of the images of the $x$ neighbours of $w$. However, $w$ can be mapped into every other vertex of $G_{2}$.

\section{Proof of Theorem 1.5}

Let $G_{1}$ and $G_{2}$ be graphs of order $n>(10 / \varepsilon)^{6}$ such that $e\left(G_{1}\right) \leqslant \alpha n, e\left(G_{2}\right) \leqslant c n^{3 / 2}$, and $\Delta\left(G_{2}\right)<n-1-\frac{\sqrt{n}}{\sqrt{2 \alpha(1-\alpha)}}$. Since $\alpha \geqslant 1 / 2$, condition (1.1) yields that $c<1 / 2$. Since the greater is $c$, the stronger is the assertion, we may assume that

$$
\frac{1}{3}<c<\frac{1}{2}
$$

Observe that, by (1.2),

$$
8(\alpha+\varepsilon) c^{2}<1-2 \varepsilon \text { and } \alpha+2 \varepsilon<1 .
$$

Let $T_{1}, \ldots, T_{t}$ be the components of $G_{1}$ that are trees (including isolated vertices) with $v\left(T_{1}\right) \leqslant \cdots \leqslant v\left(T_{t}\right)$, where we write $v(H)=|V(H)|$ for the order of a graph $H$. Let $G_{1}^{*}=G_{1}-T_{1}-\cdots-T_{t}$. In other words, let $G_{1}^{*}$ be the union of the components of $G_{1}$ containing cycles. Suppose that $G_{1}^{*}$ has exactly $\gamma n$ vertices. Then it has at least $\gamma n$ edges and hence $\gamma \leqslant \alpha$. Since $e\left(G_{1}\right) \leqslant \alpha n$,

$$
t \geqslant(1-\alpha) n
$$

It is trivial to check that the following assertion holds.

Claim 3.1. For every $1 \leqslant j<t$, we have

(a) $\sum_{i=1}^{j} v\left(T_{i}\right) \leqslant \frac{1-\gamma}{1-\alpha} j$, and

(b) $v\left(T_{j}\right) \leqslant \frac{n(1-\gamma)}{t-j+1}$.

Let $w_{1}, w_{2}, \ldots, w_{n}$ be a degenerate order of the vertices of $G_{1}$ with the additional condition that first we list vertices in $T_{1}$, then those in $T_{2}$, and so on, and we enumerate the vertices in $G_{1}^{*}$ only after having enumerated all vertices in $T_{1}, \ldots, T_{t}$. Let $u_{1}, u_{2}, \ldots, u_{n}$ be a greedy order of the vertices of $G_{2}$. Let $k_{0}^{\prime}$ be the maximal $k$ such that $\operatorname{deg}_{G_{2}(k)}\left(u_{k}\right)>\frac{(1-\alpha)^{2}}{20} n$. Since $e\left(G_{2}\right) \geqslant \sum_{i=1}^{k_{0}^{\prime}} \operatorname{deg}_{G_{2}(k)}\left(u_{k}\right)$, we have

$$
k_{0}^{\prime}<\frac{20 c}{(1-\alpha)^{2}} \sqrt{n} \leqslant \frac{10}{(1-\alpha)^{2}}(0.1 \varepsilon)^{3} n \leqslant 0.01(1-\alpha) n .
$$

Claim 3.2. For $j=1, \ldots, k_{0}^{\prime}$, there is a set $U_{j} \subset V\left(G_{2}\right)$ such that

(i) $U_{j} \supset\left\{u_{1}, \ldots, u_{j}\right\}$, 
(ii) $\left|U_{j}\right|=\sum_{i=1}^{j} v\left(T_{i}\right)$,

(iii) there exists a packing of $G_{1}\left[V\left(T_{1}\right) \cup \cdots \cup V\left(T_{j}\right)\right]$ and $G_{2}\left[U_{j}\right]$.

Proof. Suppose that the claim is proved for $j^{\prime} \leqslant j-1 \leqslant k_{0}^{\prime}-1$. Assume that the vertices of $T_{j}$ are $w_{z-y+1}, w_{z-y+2}, \ldots, w_{z}$. Let $m$ be the smallest index such that $u_{m} \notin U_{j-1}$. By the induction assumption, $m \geqslant j$. Identify $u_{m}$ with $w_{z}$ and denote $v_{0}=u_{m}$. To prove the claim, it is enough to find for every $i=1, \ldots, y-1$ a vertex $v_{i} \in V\left(G_{2}\right)-U_{j-1}-\left\{v_{0}, \ldots, v_{i-1}\right\}$ not adjacent to the vertex $v_{i^{\prime}}, i^{\prime}<i$ that was identified with a neighbour $w_{z-i^{\prime}}$ of $w_{i}$. Then we can identify $v_{i}$ with $w_{z-i}$ and continue.

Case 1: $j \leqslant 2 c \sqrt{n}$. Then by Claim 3.1(a), $\left|U_{j-1} \cup\left\{v_{0}, \ldots, v_{i-1}\right\}\right| \leqslant \frac{j}{1-\alpha}$. Since, under conditions of the theorem, $v_{i^{\prime}}$ has at least $\frac{\sqrt{n}}{\sqrt{2 \alpha}(1-\alpha)}$ non-neighbours, it has a non-neighbour in $V\left(G_{2}\right)-U_{j-1}-\left\{v_{0}, \ldots, v_{i-1}\right\}$.

Case 2: $j>2 c \sqrt{n}$. Then $\operatorname{deg}_{G_{2}(j)}\left(v_{i^{\prime}}\right) \leqslant \operatorname{deg}_{G_{2}(j)}\left(u_{j}\right) \leqslant \frac{c 1^{1.5}}{j}<n / 2$ and by Claim 3.1(a), $\left|U_{j-1} \cup\left\{v_{0}, \ldots, v_{i-1}\right\}\right|<k_{0}^{\prime} \frac{1-\gamma}{1-\alpha}$. By (3.4), the last expression is at most $0.01 n$. Again, we can choose $v_{i}$ as needed.

Let $U=U_{k_{0}^{\prime}}$ be a set provided by the claim above. We reorder the vertices $u_{1}^{\prime}, u_{2}^{\prime}, \ldots, u_{n}^{\prime}$ of $G_{2}$ as follows: first we enumerate the vertices of $U$ in any order, and then enumerate the vertices of $G_{2}-U$ in a greedy order. We will denote $k_{0}=|U|$.

Claim 3.3. $\varphi\left(G_{2}-U\right)=\sum_{v \in V\left(G_{2}-U\right)} \frac{1}{1+d_{G_{2}-U}(v)} \geqslant \frac{n}{1+2 c \sqrt{n}}$.

Proof. Let $H=G_{2}-U$. Since $\varphi$ is convex,

$$
\varphi(H) \geqslant \frac{v(H)}{1+\frac{2 e(H)}{v(H)}} .
$$

Recall that $v(H)=n-k_{0} \geqslant n-\frac{k_{0}^{\prime}(1-\gamma)}{1-\alpha}$ and $e(H) \leqslant c n \sqrt{n}-\frac{k_{0}^{\prime}(1-\alpha)^{2}}{20(1-\gamma)} n$. Thus, to prove the claim, we will verify that

$$
\frac{n-\frac{k_{0}^{\prime}}{1-\alpha}}{1+\frac{2\left(c n \sqrt{n}-\frac{k_{0}^{\prime}(1-\alpha)^{2}}{20} n\right)}{n-\frac{k_{0}^{\prime}}{1-\alpha}}} \geqslant \frac{n}{1+2 c \sqrt{n}} .
$$

Multiplying both parts of (3.5) by the product of the denominators, opening the parentheses in the left-hand side, and cancelling $n$ in both parts, we get

$$
2 c n \sqrt{n}-\frac{k_{0}^{\prime}}{1-\alpha}-\frac{2 c k_{0}^{\prime} \sqrt{n}}{1-\alpha} \geqslant \frac{2 n^{2}}{n-\frac{k_{0}^{\prime}}{1-\alpha}}\left(c \sqrt{n}-\frac{k_{0}^{\prime}(1-\alpha)^{2}}{20}\right) .
$$

Multiplying both parts of the last inequality by $n-\frac{k_{0}^{\prime}}{1-\alpha}$, cancelling $2 c n^{2} \sqrt{n}$ in both parts and dividing the rest by $\frac{-k_{0}^{\prime}}{1-\alpha}$ we obtain that (3.5) is equivalent to

$$
2 c n \sqrt{n}+\left(n-\frac{k_{0}^{\prime}}{1-\alpha}\right)(1+2 c \sqrt{n}) \leqslant 0.1 n^{2}(1-\alpha)^{3},
$$


which is weaker than

$$
1+4 c \sqrt{n} \leqslant 0.1 n(1-\alpha)^{3} .
$$

By (3.1), (1.2), and (1.3), inequality (3.6) holds.

The main difficulties of packing below are: (1) packing vertices of $G_{2}$ of very high degree; (2) packing cyclic components of $G_{1}$, (3) packing big components of $G_{1}$ that are trees, and (4) finishing the packing when there is not much freedom.

Our strategy will be the following.

Step 1: Map $V\left(T_{1} \cup \cdots \cup T_{k_{0}^{\prime}}\right)$ onto $U$.

Step 2: Find some $k_{1}, k_{0} \leqslant k_{1} \leqslant(1-\gamma-\varepsilon / 2) n+\frac{1}{1-\alpha}$ so that the maximum degree of $G_{2}\left(k_{1}+1\right)$ is moderate.

Step 3: Map the vertices of $G_{1}^{*}$ into (the complement of) $G_{2}\left(k_{1}+1\right)$.

Step 4: Map the vertices of $T_{t}, T_{t-1}, \ldots, T_{1+\lfloor 3 n(1-\alpha) / 4\rfloor}$ into some of the remaining free vertices of $G_{2}$.

Step 5: Complete the packing by arranging the vertices of the remaining tree-components of $G_{1}$ in the rest of $G_{2}$.

Step 1 will take care of difficulty (1), Steps 2 and 3 handle (2), and at Step 4 we overcome (3).

We can complete Step 1 by Claim 3.2. Note that $G_{2}$ with the enumeration $u_{1}^{\prime}, \ldots, u_{n}^{\prime}$ satisfies condition (ii) of Lemma 2.2 and $k_{0}$ satisfies the restrictions in this lemma. Suppose that condition (i) fails for $G_{2}$ and $k_{0}$, i.e., that

$$
e\left(G_{2}-U\right)>e\left(G_{2}\right)\left(1-\frac{2 k_{0}(\alpha+\varepsilon)}{n(\alpha-\gamma+\varepsilon / 2)}\right) .
$$

Then the number $\tilde{e}(U)$ of edges in $G_{2}$ incident with $U$ is less than

$$
c n^{3 / 2} \frac{2 k_{0}(\alpha+\varepsilon)}{n(\alpha-\gamma+\varepsilon / 2)}<c \sqrt{n} \frac{2 k_{0}}{\alpha-\gamma+\varepsilon / 2} .
$$

On the other hand, by the definition of $k_{0}^{\prime}, \tilde{e}(U)>k_{0}^{\prime} \frac{(1-\alpha)^{2}}{20} n$, and by Claims 3.1 and 3.2, $k_{0}^{\prime} \geqslant k_{0} \frac{1-\alpha}{1-\gamma}$. Thus if condition (i) fails for $G_{2}$ and $k_{0}$, then

$$
k_{0} \frac{1-\alpha}{1-\gamma} \frac{(1-\alpha)^{2}}{20} n<c \sqrt{n} \frac{2 k_{0}(1-\gamma)}{\alpha-\gamma+\varepsilon / 2}
$$

and hence

$$
\begin{aligned}
\sqrt{n} & <\frac{40 c(1-\gamma)}{(1-\alpha)^{3}(\alpha-\gamma+\varepsilon / 2)}<\frac{20((1-\alpha)+(\alpha-\gamma))}{(1-\alpha)^{3}(\alpha-\gamma+\varepsilon / 2)} \\
& <\frac{20}{(1-\alpha)^{2}(\alpha-\gamma+\varepsilon / 2)}+\frac{20}{(1-\alpha)^{3}} \\
& \leqslant \frac{20}{(1-\alpha)^{2} \varepsilon / 2}+\frac{20}{(1-\alpha)^{3}} \leqslant \frac{60}{(1-\alpha)^{2} \varepsilon}<\frac{15}{\varepsilon^{3}}
\end{aligned}
$$

This contradicts (1.3).

Therefore, $G_{2}$ with the enumeration $u_{1}^{\prime}, \ldots, u_{n}^{\prime}$ satisfies the conditions of Lemma 2.2. This lemma implies that there is an index $k_{0} \leqslant k_{1} \leqslant(1-\gamma-\varepsilon / 2) n$ such that the maximal 
degree $D=\Delta(H)$ of the graph $H=G_{2}\left(k_{1}+1\right)$ satisfies

$$
D \leqslant \frac{2 c\left(n-k_{1}\right)(\alpha+\varepsilon)}{\sqrt{n}(\alpha-\gamma+\varepsilon / 2)} .
$$

This completes Step 2. Note that the right-hand side of (3.7) is at most $\frac{4 c(\alpha+\varepsilon)}{\varepsilon} \sqrt{n}$ and hence (3.7) together with (3.2) yields

$$
D \leqslant \frac{4 c(\alpha+\varepsilon)}{\varepsilon} \sqrt{n} \leqslant \frac{\sqrt{n}}{2 c \varepsilon} \leqslant \frac{3 \sqrt{n}}{2 \varepsilon} .
$$

Also, by Theorem 2.1 and Claim 3.3, for

$$
\ell=\left\lceil\frac{n}{2 c \sqrt{n}+1}\right\rceil
$$

the set $L=\left\{u_{n-\ell+1}^{\prime}, u_{n-\ell+2}^{\prime}, \ldots, u_{n}^{\prime}\right\}$ of the last $\ell$ vertices of $G_{2}$ forms an independent set in $G_{2}$.

Now, we identify the last $\ell$ vertices of $G_{1}$ with vertices in $L$. Since $L$ is an independent set, this identification is 'legal' so far: no edge of $G_{1}$ is identified with an edge of $G_{2}$. If $w_{n-\ell}$ is not in $G_{1}^{*}$, then Step 3 is done, otherwise we continue as follows. We place the vertices $w_{n-\ell}, w_{n-\ell-1}, \ldots, w_{(1-\gamma) n+1}$ one by one into the rest of $G_{2}$, the 'middle' of $G_{2}$, namely $M=V\left(G_{2}\left(k_{1}+1\right)\right)-L$. We show now that all these vertices can be placed into $M$ to give us a packing of $G_{1}^{*}$ into (the complement of) $G_{2}$.

Suppose that we have placed the vertices $w_{n-\ell}, w_{n-\ell-1}, \ldots, w_{n-j+1}$ into $M$, and the next vertex to be placed, $w_{n-j}$, has $x$ neighbours $w_{h}$ with $h>n-j$. Since $w_{1}, w_{2}, \ldots, w_{n}$ is a degenerate order of the vertices of $G_{1}$, the subgraph $G_{1}(n-j)$ has minimal degree $x$. Furthermore, as $G_{1}^{*}$ has $\gamma n$ vertices, we find that

$$
j x+2(\gamma n-j) \leqslant 2 e\left(G_{1}\right) \leqslant 2 \alpha n,
$$

and so

$$
x \leqslant 2+2(\alpha-\gamma) n / j .
$$

By Claim 2.3, we have a legal placement for $w_{n-j}$ provided that

$$
n-k_{1}-j-x D>0 .
$$

Thus, to complete Step 3, it suffices to check that (3.11) holds.

Suppose that (3.11) is false. Then, by (3.10) and (3.7), we have

$$
n-k_{1}-j \leqslant D\left(2+\frac{2(\alpha-\gamma) n}{j}\right)<2 D+\frac{2 c(\alpha+\varepsilon)\left(n-k_{1}\right) 2(\alpha-\gamma) n}{\sqrt{n}(\alpha-\gamma+0.5 \varepsilon) j} .
$$

Add $j$ to both parts of (3.12) and divide both parts by $n-k_{1}$. Taking into account (3.8) and the fact that $k_{1} \leqslant n(1-\gamma-\varepsilon / 2)$, we get

$$
1<\frac{2 D+j}{n-k_{1}}+\frac{4 c(\alpha+\varepsilon)(\alpha-\gamma) n}{\sqrt{n}(\alpha-\gamma+0.5 \varepsilon) j} \leqslant \frac{3 \sqrt{n} / \varepsilon+j}{n(\gamma+0.5 \varepsilon)}+\frac{4 c(\alpha+\varepsilon) \sqrt{n}}{j} .
$$

Consider the right-hand side of (3.13) as the function $f(j)$. This is a convex function of $j$ (when other parameters are fixed). Since $\ell<j \leqslant \gamma n$, by (3.9), it is enough to check that 
$f(j) \leqslant 1$ for $j=\frac{n}{2 c \sqrt{n}+1}$ and $j=\gamma n$. Taking (1.1) into account, we get

$$
\begin{aligned}
f\left(\frac{n}{2 c \sqrt{n}+1}\right) & \leqslant \frac{3 \sqrt{n} / \varepsilon+2 \sqrt{n}}{n(\gamma+0.5 \varepsilon)}+\frac{4 c(\alpha+\varepsilon) \sqrt{n}(1+2 c \sqrt{n})}{n} \\
& \leqslant \frac{\frac{3}{\varepsilon}+2}{0.5 \varepsilon \sqrt{n}}+\frac{4 c(\alpha+\varepsilon)}{\sqrt{n}}+8 c^{2}(\alpha+\varepsilon) .
\end{aligned}
$$

By (3.2) and (1.3), the last expression is at most

$$
\left(\frac{3}{\varepsilon}+2\right)(0.1 \varepsilon)^{2}+\frac{(0.1 \varepsilon)^{3}}{2 c}+1-2 \varepsilon<\frac{3 \varepsilon}{100}+\frac{\varepsilon^{2}}{50}+\frac{2 \varepsilon^{3}}{1000}+1-2 \varepsilon<1 .
$$

Now,

$$
f(\gamma n)=\frac{3}{\varepsilon \sqrt{n}(\gamma+0.5 \varepsilon)}+\frac{\gamma}{\gamma+0.5 \varepsilon}+\frac{4 c(\alpha+\varepsilon)}{\gamma \sqrt{n}} .
$$

If $\gamma \geqslant 0.1 \varepsilon^{2}$ then, by (3.2) and (1.3), the last expression is at most

$$
\frac{6}{\varepsilon^{2} \sqrt{n}}+\frac{1}{1+0.5 \varepsilon}+\frac{5}{c \varepsilon^{2} \sqrt{n}} \leqslant \frac{6 \varepsilon}{1000}+1-\frac{0.5 \varepsilon}{1+0.5 \varepsilon}+\frac{15 \varepsilon}{1000}<1 .
$$

Suppose that $\gamma<0.1 \varepsilon^{2}$. Since $\gamma n>\ell$, we obtain by (3.9) and (3.2) that

$$
\begin{aligned}
f(\gamma n) & \leqslant \frac{6}{\varepsilon^{2} \sqrt{n}}+\frac{0.1 \varepsilon^{2}}{0.1 \varepsilon^{2}+0.5 \varepsilon}+\frac{4 c(\alpha+\varepsilon) \sqrt{n}(1+2 c \sqrt{n})}{n} \\
& \leqslant \frac{6}{\varepsilon^{2} \sqrt{n}}+\frac{\varepsilon}{5}+\frac{4 c(\alpha+\varepsilon)}{\sqrt{n}}+8 c^{2}(\alpha+\varepsilon) \\
& \leqslant \frac{6}{\varepsilon^{2} \sqrt{n}}+\frac{\varepsilon}{5}+\frac{1}{2 c \sqrt{n}}+(1-2 \varepsilon) \leqslant \frac{8 \varepsilon}{1000}+1-1.8 \varepsilon<1 .
\end{aligned}
$$

This finishes Step 3.

Let $G_{2}^{\prime}$ denote the subgraph of $G_{2}$ induced by the vertices not used as the images of vertices in $G_{1}^{*}$, and in $T_{1}, \ldots, T_{k_{0}^{\prime}}$. Then by (3.4) and Claim 3.1,

$$
\begin{gathered}
n_{2}^{\prime}=\left|V\left(G_{2}^{\prime}\right)\right| \geqslant(1-\gamma) n-k_{0} \geqslant(1-\gamma)\left(n-\frac{k_{0}^{\prime}}{1-\alpha}\right) \\
\geqslant(1-\gamma)\left(n-\frac{0.01(1-\alpha) n}{1-\alpha}\right) \geqslant 0.99(1-\alpha) n .
\end{gathered}
$$

By the definition of $k_{0}^{\prime}$, the maximum degree $D^{\prime}$ of $G_{2}^{\prime}$ is at most $\frac{(1-\alpha)^{2}}{20} n$. Since the subgraph $G_{1}^{\prime}$ of $G_{1}$ induced by $V\left(T_{t} \cup T_{t-1} \cup \cdots \cup T_{1+\lfloor 3 n(1-\alpha) / 4\rfloor}\right)$ is 1-degenerate, we can apply Claim 2.3 with $x=1$. The claim implies that we can complete Step 4 provided

$$
n_{2}^{\prime}>D^{\prime}+\left|V\left(G_{1}^{\prime}\right)\right|
$$

Applying (3.4), we have

$$
n_{2}^{\prime}-\left|V\left(G_{1}^{\prime}\right)\right| \geqslant \sum_{i=k_{0}^{\prime}+1}^{\lfloor 3 n(1-\alpha) / 4\rfloor} v\left(T_{i}\right) \geqslant \frac{3(1-\alpha)}{4} n-k_{0}^{\prime}-1>0.74(1-\alpha) n-1>\frac{2(1-\alpha)}{3} n .
$$

Taking into account that $D^{\prime} \leqslant \frac{(1-\alpha)^{2}}{20} n$, we get (3.15). 
Remarks. (1) Any vertex in a tree could be made the last vertex in a degenerate order. In particular, we can make the last a vertex of maximum degree.

(2) Packing each tree, we can start from identifying a vertex of the highest degree in this tree with an available vertex of the smallest degree in $G_{2}$.

Finally, let $G_{2}^{\prime \prime}$ denote the subgraph of $G_{2}$ induced by the vertices not yet used as the images of vertices in $G_{1}$. Then, as in the previous paragraph,

$$
n_{2}^{\prime \prime}=\left|V\left(G_{2}^{\prime \prime}\right)\right|>\frac{2(1-\alpha)}{3} n
$$

and $\Delta\left(G_{2}^{\prime \prime}\right) \leqslant D^{\prime} \leqslant \frac{(1-\alpha)^{2}}{20} n$. Let $G_{1}^{\prime \prime}=T_{k_{0}^{\prime}+1} \cup T_{k_{0}^{\prime}+2} \cup \cdots \cup T_{\lfloor 3 n(1-\alpha) / 4\rfloor}$. By Claim 3.1(b), the maximum degree $D_{1}$ of $G_{1}^{\prime \prime}$ is less than $\frac{4}{1-\alpha}$. Therefore,

$$
D_{1} \cdot D^{\prime} \leqslant \frac{4}{1-\alpha} \cdot \frac{(1-\alpha)^{2}}{20} n=\frac{(1-\alpha)}{5} n<\frac{n_{2}^{\prime \prime}}{2} .
$$

Thus, by Theorem 1.1, $G_{1}^{\prime \prime}$ and $G_{2}^{\prime \prime}$ pack. This proves Theorem 1.5.

\section{Packing many graphs}

In this section, we use Theorem 1.5 to show that one can pack many graphs if each of these graphs has at most $\alpha n$ edges. First, we look again into the proof of Theorem 1.5.

Lemma 4.1. Let $\alpha, c, n$ and $G_{1}$ and $G_{2}$ satisfy the conditions of Theorem 1.5. Let $H=G_{1} \cup$ $G_{2}$ be the graph with $V(H)=V\left(G_{1}\right)=V\left(G_{2}\right), E(H)=E\left(G_{1}\right) \cup E\left(G_{2}\right)$ obtained by packing $G_{1}$ and $G_{2}$ as described in the proof of Theorem 1.5. Then $\Delta(H) \leqslant \max \{\alpha n+0.04(1-$ $\left.\alpha) n, \Delta\left(G_{2}\right)+2 /(1-\alpha)\right\}$.

Proof. Suppose that the lemma is false. Then there is a vertex $v$ with $\operatorname{deg}_{H}(v)>$ $\max \left\{\alpha n, \Delta\left(G_{2}\right)\right\}+2 /(1-\alpha)$. We may assume that $v$ is the result of identifying $w_{i} \in V\left(G_{1}\right)$ with $u_{j} \in V\left(G_{2}\right)$.

Case 1: $\operatorname{deg}_{G_{2}}\left(u_{j}\right)>0.5 \alpha n+2$. If $j>k_{0}$, then by (3.4) and the definition of $k_{0}^{\prime}$,

$$
\operatorname{deg}_{G_{2}}\left(u_{j}\right) \leqslant k_{0}^{\prime}+\frac{(1-\alpha)^{2}}{20} n<0.01(1-\alpha) n+\frac{(1-\alpha)^{2} n}{20}<0.04(1-\alpha) n \leqslant 0.02 n,
$$

a contradiction. Therefore, $j \leqslant k_{0}$. Hence, $w_{i} \in V\left(T_{1} \cup \cdots \cup T_{k_{0}^{\prime}}\right)$ and $\operatorname{deg}_{G_{1}}\left(w_{i}\right) \leqslant\left|V\left(T_{k_{0}^{\prime}}\right)\right|-1$. By Claim 3.1(b), $\left|V\left(T_{k_{0}^{\prime}}\right)\right| \leqslant \frac{n}{n(1-\alpha)-k_{0}^{\prime}+1}$. In view of (3.4),

$$
n(1-\alpha)-k_{0}^{\prime}+1>n(1-\alpha)-0.01 n(1-\alpha)=0.99 n(1-\alpha) .
$$

It follows that $\operatorname{deg}_{G_{1}}\left(w_{i}\right)<\frac{1}{0.99(1-\alpha)}$ and the lemma holds.

Case 2: $\operatorname{deg}_{G_{1}}\left(w_{i}\right)>0.5 \alpha n+2$. Since $e\left(G_{1}\right)=\alpha n$, there is only one vertex in $G_{1}$ with this property. Furthermore, with such a large degree, $w_{i}$ is either in $V\left(G_{1}^{*}\right)$, or in $V\left(T_{t}\right)$. In either case, $u_{j} \notin U$, and by $(4.1), \operatorname{deg}_{G_{2}}\left(u_{j}\right) \leqslant 0.04(1-\alpha) n$. This proves the lemma. 
Now we are ready to prove Theorem 1.8 .

Proof. Recall that $m=\left[0.25 \sqrt{n / \alpha^{3}}\right]$. We will prove by induction on $k$, that for $k=$ $1, \ldots, m$, there is a packing of $H_{1}, \ldots, H_{k}$ such that the maximal degree, $\Delta\left(F_{k}\right)$, of the obtained graph $F_{k}=H_{1} \cup \cdots \cup H_{k}$ is at most $(1-0.96(1-\alpha)) n+2(k-2) /(1-\alpha)$.

For $k=1$, the statement reduces to $\Delta\left(H_{1}\right) \leqslant \alpha n+0.04 n-2 /(1-\alpha)$. By $(1.4), 0.04 n-$ $2 /(1-\alpha) \geqslant 0$ which proves the base case.

Suppose that the theorem is proved for some $k \leqslant m-1$. Let us check that Theorem 1.5 and Lemma 4.1 hold for our $\alpha$ and $n, c=e\left(F_{k}\right) / n^{3 / 2}, \varepsilon=0.25(1-\alpha), G_{1}=H_{k+1}$, and $G_{2}=F_{k}$. Indeed, since $k \leqslant m-1$, we have

$$
e\left(F_{k}\right) \leqslant k \alpha n \leqslant(m-1) \alpha n<\frac{\alpha n \sqrt{n}}{4 \alpha^{3 / 2}}
$$

and hence $c \leqslant 0.25 / \sqrt{\alpha}$. Therefore, $8 c^{2} \alpha \leqslant 1 / 2$, which yields (1.1) and (1.2). Now, (1.3) follows from (1.4). By the inductive assumption,

$$
\begin{aligned}
\Delta\left(G_{2}\right) & \leqslant(1-0.96(1-\alpha)) n+\frac{2(k-2)}{1-\alpha} \leqslant n-\frac{2}{1-\alpha}-\left(0.96(1-\alpha) n-\frac{2(m-2)}{1-\alpha}\right) \\
& \leqslant n-2-\left(0.96(1-\alpha) n-\frac{2 \sqrt{n}}{4(1-\alpha) \alpha^{1.5}}\right) \leqslant n-2-\left(0.96(1-\alpha) n-\frac{\sqrt{2 n}}{1-\alpha}\right) .
\end{aligned}
$$

Observe that

$$
0.96(1-\alpha) n>0.96 \sqrt{n} \frac{50^{3}}{(1-\alpha)^{2}}>\frac{100 \sqrt{n}}{1-\alpha}
$$

and hence

$$
\Delta\left(G_{2}\right) \leqslant n-2-\frac{50 \sqrt{n}}{1-\alpha} .
$$

Thus, the conditions of Theorem 1.5 are satisfied, and by Lemma 4.1 we can pack $H_{k+1}$ and $F_{k}$ so that the maximum degree $\Delta\left(F_{k+1}\right)$ of the resulting graph $F_{k+1}=F_{k} \cup H_{k+1}$ exceeds $(1-0.96(1-\alpha)) n+2(k-2) /(1-\alpha)$ by at most $2 /(1-\alpha)$. This proves the theorem.

\section{Sparse graphs that do not pack}

We will construct some series of pairs of sparse graphs that do not pack. We start from a simple series and then elaborate it.

Let $G_{1}=G_{1}(n, 2)$ be a forest on $n$ vertices whose components are stars $S_{1}$ and $S_{2}$ of degree at most $\left\lceil\frac{n}{2}\right\rceil$. By $s_{1}$ and $s_{2}$ we denote the centres of these stars.

Let $W=\left\{w_{1}, w_{2}, w_{3}\right\}$ and $U$ be a set disjoint from $W$ with $|U|=n-3$ partitioned into subsets $U_{1}, U_{2}$, and $U_{3}$ of about the same cardinality. We define $G_{2}=G_{2}(n, 1,2)$ as follows. Let $V_{2}=V\left(G_{2}\right)=W \cup U$ and $E_{2}=\left\{w_{i} w_{j} \mid 1 \leqslant i<j \leqslant 3\right\} \cup \bigcup_{i=1}^{3}\left\{u w_{i}, u w_{i+1} \mid u \in U_{i}\right\}$ (we sum the indices modulo 3 ). The graph $G_{2}$ possesses the property that every two vertices have a common neighbour and the maximum degree of $G_{2}$ is $\lceil 2 n / 3\rceil$. Furthermore, $G_{2}$ is 2-degenerate, i.e., very sparse.

Suppose that $G_{1}(n, 2)$ and $G_{2}(n, 1,2)$ pack, i.e., that there is an edge-disjoint placement $f$ of the vertex set $V_{1}$ of $G_{1}$ onto $V_{2}$. Let $t_{1}=f\left(s_{1}\right)$ and $t_{2}=f\left(s_{2}\right)$. By the previous 
paragraphs, $t_{1}$ and $t_{2}$ have a common neighbour, say, $t_{0}$, in $G_{2}$. Then the vertex $s_{0}$ in $G_{1}$ with $f\left(s_{0}\right)=t_{0}$ cannot be adjacent to any of $s_{1}$ and $s_{2}$. This contradicts the definition of $G_{1}$. Thus $G_{1}$ and $G_{2}$ do not pack.

Note that this example disproves Conjecture 1.6 and shows that to extend the statement of Theorem 1.5 even to $\alpha=1$, one needs to impose sufficiently stricter conditions on the maximum degree of $G_{2}$. The maximum of maximum degrees of $G_{1}$ and $G_{2}$ is $\lceil 2 n / 3\rceil$. Below, we elaborate the above example to make this maximum less by making greater the average degree of $G_{2}$.

Let $G_{1}=G_{1}(n, k)$ be a forest on $n$ vertices whose $k$ components are stars $S_{1}, \ldots, S_{k}$ of degree at most $\left\lceil\frac{n}{k}\right\rceil$. By $s_{1}, \ldots, s_{k}$ we denote the centres of these stars.

Let $q$ be a prime power. For a nonnegative integer $d$, let $q_{d}=\frac{q^{d+1}-1}{q-1}$. In particular, $q_{0}=1$ and $q_{1}=q+1$. Suppose that $n>q^{k+1}$. To construct $G_{2}=G_{2}(n, q, k)$, consider a $k$-dimensional projective space $W$ over the field $G F_{q}$. It has $q_{k}$ points and $q_{k}$ hyperplanes. Let $U$ be a set of $n-q_{k}$ vertices partitioned into $q_{k}$ sets $U_{1}, \ldots, U_{q_{k}}$ with $\left|U_{i}\right| \leqslant\left\lceil\frac{n}{q_{k}}\right\rceil-1$ for all $i$. Let $\left\{H_{1}, \ldots, H_{q_{k}}\right\}$ be a list of all hyperplanes in $W$. The graph $G_{2}=G_{2}(n, q, k)$ has the vertex set $V_{2}=W \cup U$ and the edge set

$$
E_{2}=\left\{w_{1} w_{2} \mid w_{1} \in H_{1}, w_{2} \in W, w_{1} \neq w_{2}\right\} \cup \bigcup_{i=1}^{q_{k}}\left\{w u \mid w \in H_{i}, u \in U_{i}\right\} .
$$

Claim 5.1. If $n>q^{k+1}$, then

(a) $G_{2}(n, q, k)$ is $q_{k-1}$-degenerate,

(b) $\left|E_{2}\right|<q_{k-1} n$,

(c) the maximum degree of $G_{2}(n, q, k)$ is at most $\frac{n}{q}+q_{k}$.

Proof. Order the vertices of $G_{2}$ so that first we list the vertices in $U$, then the vertices in $W-H_{1}$, and finally the points of $H_{1}$. Then every vertex $v$ has at most $q_{k-1}$ neighbours following $v$ in this order. This proves (a). Note that (a) yields (b).

To check (c), observe that every vertex in $U$ has degree $q_{k-1}$. Every point of a $k$ dimensional projective space over $G F_{q}$ is contained in $q_{k-1}$ hyperplanes. Therefore, every $w \in W$ is adjacent to at most $q_{k-1}\left(\left\lceil\frac{n}{q_{k}}\right\rceil-1\right)<\frac{n}{q}$ vertices in $U$. Since $|W|=q_{k}$, this proves (c).

Claim 5.1 implies that for fixed $q$ and $k, G_{2}(n, q, k)$ has linear in $n$ number of edges. Furthermore, if $n>q \cdot q_{k}$, then the maximum degree of $G_{2}$ is less than $\frac{2 n}{q}$. Thus, for every $k$ and any prime power $q \geqslant 2 k$, if $n>q \cdot q_{k}$, then both $G_{1}(n, k)$ and $G_{2}(n, q, k)$ have maximum degree at most $n / k$.

Claim 5.2. If $n>q \cdot q_{k}$, then $G_{1}(n, k)$ and $G_{2}(n, q, k)$ do not pack.

Proof. Suppose that there exists a packing of $G_{1}(n, q)$ and $G_{2}(n, q, k)$, i.e., that there is an edge-disjoint placement $f$ of the vertex set $V_{1}$ of $G_{1}$ onto $V_{2}$. Let $t_{j}=f\left(s_{j}\right)$ for $j=1, \ldots, k$. By the definition of $G_{2}$, the neighbourhood of every of $t_{j}$ contains some $H_{i(j)}$ (if $t_{j} \in H_{1}$, then it contains many $\left.H_{i}\right)$. Suppose that the set $T=\left\{t_{1}, \ldots, t_{k}\right\}$ contains exactly $r$ vertices 
of $H_{1}$. Since any $k-r$ hyperplanes of $W$ have a common $r$-dimensional subspace, the neighbourhoods in $G_{2}$ of the remaining $k-r$ elements of $T$ have at least $q_{r}$ vertices in common. Since $q_{r}>r$ and vertices of $H_{1}$ are adjacent to every vertex in $W$, there exists a common neighbour $t_{0} \in W$ of all vertices in $T$. But then the vertex $s_{0}=f^{-1}\left(t_{0}\right)$ cannot be adjacent in $G_{1}$ to any of $s_{1}, \ldots, s_{k}$. This contradicts the definition of $G_{1}$.

These two claims prove Theorem 1.7.

\section{Acknowledgement}

We are grateful to the referees for their helpful comments.

\section{References}

[1] Aigner, M. and Brandt, S. (1993) Embedding arbitrary graphs of maximum degree two. J. London Math. Soc. 48 39-51.

[2] Alon, N. and Fischer, E. (1996) 2-factors in dense graphs. Discrete Math. 152 13-23.

[3] Bollobás, B. (1978) Extremal Graph Theory, Academic Press, London/New York.

[4] Bollobás, B. and Eldridge, S. E. (1976) Maximal matchings in graphs with given maximal and minimal degrees. Congressus Numerantium XV 165-168.

[5] Bollobás, B. and Eldridge, S. E. (1978) Packing of graphs and applications to computational complexity. J. Combin. Theory Ser. B 25 105-124.

[6] Brandt, S. (1995) An extremal result for subgraphs with few edges. J. Combin. Theory Ser. B 64 288-299.

[7] Caro, Y. (1979) New results on the independence number. Technical Report, University of Tel Aviv, Israel.

[8] Caro, Y. and Tuza, Z. (1991) Improved lower bounds on $k$-independence. J. Graph Theory 15 99-107.

[9] Catlin, P. A. (1974) Subgraphs of graphs I. Discrete Math. 10 225-233.

[10] Catlin, P. A. (1976) Embedding subgraphs and coloring graphs under extremal degree conditions. PhD Thesis, Ohio State University, Columbus, $\mathrm{OH}$.

[11] Csaba, B., Shokoufandeh, A. and Szemerédi, E. (2003) Proof of a conjecture of Bollobás and Eldridge for graphs of maximum degree three. Combinatorica 23 35-72.

[12] Hajnal, A. and Szemerédi, E. (1970) Proof of a conjecture of Erdős. In Combinatorial Theory and its Applications, Vol. II (P. Erdős, A. Rényi and V. T. Sós, eds), North-Holland, pp. 601-603.

[13] Sauer, N. and Spencer, J. (1978) Edge disjoint placement of graphs. J. Combin. Theory Ser. B 25 295-302.

[14] Wei, V. K. (1981) A lower bound on the stability number of a simple graph. Technical Memorandum TM 81-11217-9, Bell Laboratories.

[15] Wozniak, M. (1997) Packing of graphs. Dissertationes Math. 362 1-78.

[16] Yap, H. P. (1988) Packing of graphs: A survey. Discrete Math. 72 395-404. 